\title{
Critical role of stearoyl-CoA desaturase-1 (SCD1) in the onset of diet-induced hepatic insulin resistance
}

\author{
Roger Gutiérrez-Juárez,, Alessandro Pocai, ${ }^{1}$ Claudia Mulas, ${ }^{1}$ Hiraku Ono, ${ }^{1}$ \\ Sanjay Bhanot, ${ }^{2}$ Brett P. Monia, ${ }^{2}$ and Luciano Rossetti' \\ 1Department of Medicine, Department of Molecular Pharmacology, and Diabetes Research Center, \\ Albert Einstein College of Medicine, Bronx, New York, USA. ${ }^{2}$ SIS Pharmaceuticals, Carlsbad, California, USA.
}

\begin{abstract}
Stearoyl-CoA desaturase-1 (SCD1) catalyzes the synthesis of monounsaturated fatty acids from saturated fatty acids. Mice with a targeted disruption of $S c d 1$ gene locus are lean and display increased insulin sensitivity. To examine whether Scd1 activity is required for the development of diet-induced hepatic insulin resistance, we used a sequence-specific antisense oligodeoxynucleotide (ASO) to lower hepatic Scd1 expression in rats and mice with diet-induced insulin resistance. Treatment of rats with Scd1 ASO markedly decreased liver Scd1 expression $(-80 \%)$ and total Scd activity $(-50 \%)$ compared with that in rats treated with scrambled ASO (control). Insulin clamp studies revealed severe hepatic insulin resistance in high-fat-fed rats and mice that was completely reversed by 5 days of treatment with Scd1 ASO. The latter treatment decreased glucose production (by $\sim 75 \%$ ), gluconeogenesis, and glycogenolysis. Downregulation of Scd1 also led to increased Akt phosphorylation and marked decreases in the expression of glucose-6-phosphatase (Glc-6-Pase) and phosphoenolpyruvate carboxykinase (PEPCK). Thus, Scd1 is required for the onset of diet-induced hepatic insulin resistance.
\end{abstract}

\section{Introduction}

The metabolic syndrome is a complex of metabolic alterations whose landmarks include obesity, hyperlipidemia, hepatic steatosis, and insulin resistance (1). Diets rich in both simple sugars and saturated fat are regarded as causative factors in the development of insulin resistance in laboratory animals and human populations $(2-8)$. Severe hepatic insulin resistance is indeed among the earliest changes seen with short-term overfeeding $(\mathrm{OF})$ in rats $(9,10)$.

Alterations in systemic and cellular lipid trafficking are postulated to play a critical role in the development of diet-induced insulin resistance (11-19). However, the biochemical and signaling steps responsible for this tight association remain to be delineated. Various genetic models designed to manipulate cellular lipid metabolism display resistance to diet-induced insulin resistance, often accompanied by diminished fat accumulation (20-23). Among the biochemical steps frequently associated with improved insulin sensitivity is the decreased hepatic activity/expression of stearoylCoA desaturase-1 (SCD1) $(22,24,25)$. Can the decrease in SCD1 activity account for enhanced insulin action? Mice carrying a naturally occurring null allele of Scd1 (asebia mice) as well as mice with a targeted disruption of Scd1 locus display a lean phenotype with increased energy expenditure and enhanced insulin sensitivity (24, 26). Similar improvements in energy balance and metabolism are also induced by long-term (10 weeks) antisense-mediated down-

Nonstandard abbreviations used: ACC, acetyl-CoA carboxylase; AMPK, AMP kinase; ASO, antisense oligodeoxynucleotide; CYP7A1, cholesterol hydroxylase $7 \alpha$; FAS, fatty acid synthase; Glc-6-Pase, glucose-6-phosphatase; HF, high fat; IR $\beta$, insulin receptor $\beta$ subunit; IRS1, insulin receptor substrate 1 ; LCFA, long-chain fatty acyl$\mathrm{CoA}$; OF, overfed, overfeeding; $\mathrm{p}$, phosphorylated; PEPCK, phosphoenolpyruvate carboxykinase; PTP1B, protein tyrosine phosphatase 1B; Q-PCR, quantitative real-time PCR; SC, standard chow; Scd1, stearoyl-CoA desaturase-1; TG, triglycerides.

Conflict of interest: S. Bhanot and B.P. Monia are employed by ISIS Pharmaceuticals and declare that they have a financial interest in the development of SCD1 antisense inhibitors as therapeutic agents.

Citation for this article: J. Clin. Invest. 116:1686-1695 (2006). doi:10.1172/JCI26991. regulation of Scd1 expression in high-fat-fed (HF-fed) mice (27). Unrestrained Scd 1 activity in ob/ob mice has been implicated in the metabolic abnormalities produced by leptin deficiency (26). In fact, ob/ob mice carrying a null allele of Scd1 displayed a significant attenuation of obesity, of hyperinsulinemia, and of hepatic steatosis (26). Since prolonged SCD1 deficiency and leptin treatment lead to decreased fat mass and tissue lipid accumulation as well as improvements in glucose tolerance and insulin action, it is not clear whether SCD1 deficiency has a direct impact on insulin sensitivity or results in improved insulin action via the associated changes in body composition and tissue lipid content.

In order to examine whether SCD1 activity is required for the development of diet-induced hepatic insulin resistance, we rapidly lowered hepatic Scd 1 expression in adult rats, using a specific antisense oligodeoxynucleotide (ASO) directed against Scd1 mRNA. Rats were then placed on a lard-enriched diet known to rapidly induce severe hepatic insulin resistance. Short-term knockdown of Scd1 expression did not alter food intake and body weight gain, but it completely restored insulin's ability to suppress hepatic glucose production, gluconeogenesis, and glycogenolysis.

\section{Results}

In mammalian liver, SCD1 is the main enzyme responsible for the conversion of the saturated fatty acyl-CoAs, stearoyl-CoA (18:0) and palmitoyl-CoA (16:0), to their respective monounsaturated fatty acyl-CoAs, oleyl-CoA (18:1) and palmitoleyl-CoA (16:1) (Figure 1A). Here we asked whether a diet enriched in saturated fatty acids can still induce hepatic insulin resistance when the hepatic expression and activity of SCD1 is markedly curtailed.

Treatment with Scd1 ASO decreases liver Scd1 expression and activity. Following 2 injections of Scd1 ASO over 5 days (Figure 1B), liver Scd 1 mRNA was decreased by $80 \%$, as determined by quantitative real-time PCR (Q-PCR). Similarly, total SCD activity was decreased by approximately $50 \%$ in the liver of rats treated with Scd1 ASO 
A

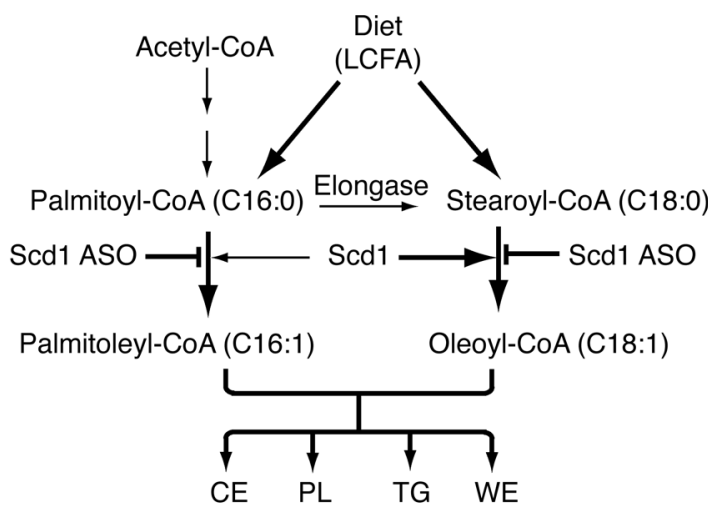

B Clamp catheters

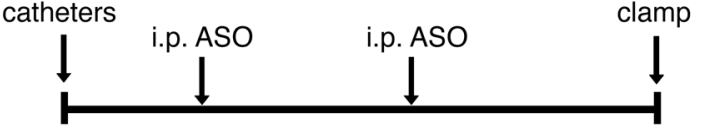

Day -2

Day 0

Day 5
C
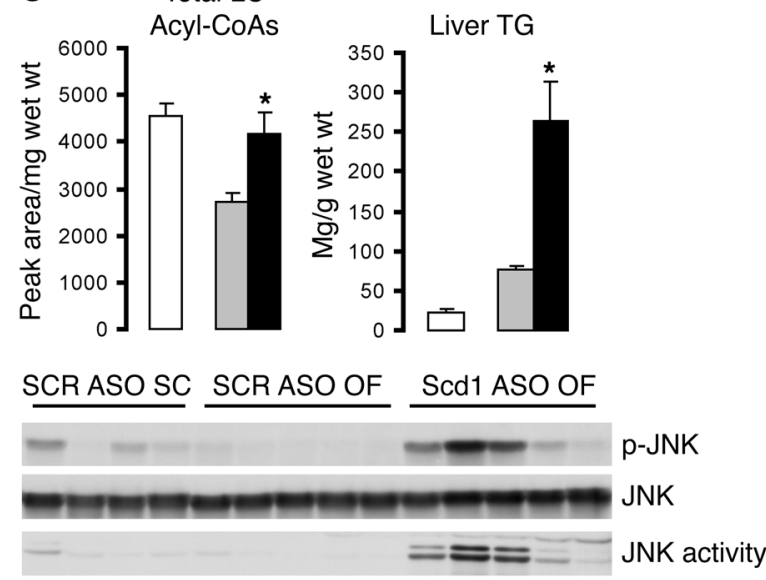

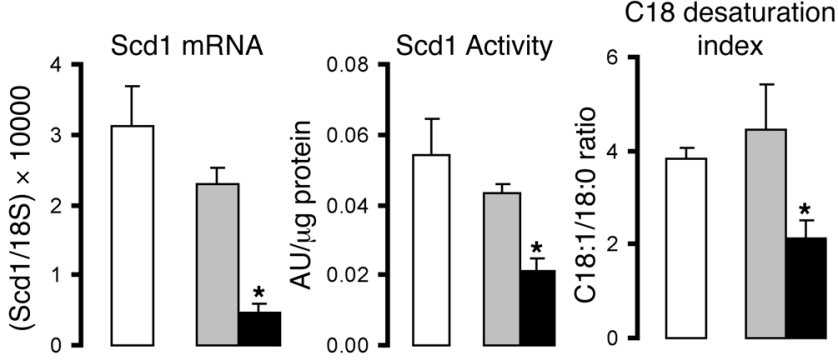

HF diet

Figure 1

Systemic administration of Scd1 ASO downregulates liver Scd1 expression and activity in OF rats. (A) Schematic of Scd1 role in lipid synthesis. CE, cholesteryl esters; PL, phospholipids; WE, wax esters. (B) Protocol for Scd1 ASO delivery (top panel). Rats received an i.p. injection of either a control ASO (SCR ASO) or Scd1 ASO (100 mg/kg of body weight) on day 0; on day 3, the animals were switched to a lard-enriched diet (HF) and received a second injection of ASOs; lastly, on day 5, an insulin clamp procedure was performed. The livers of OF animals treated with Scd1 ASO (black bars) displayed an 80\% decrease in Scd1 mRNA and a 50\% decrease in both Scd1 activity and C18 desaturation index. OF (gray bars) did not modify any of these parameters when compared with SC rats (white bars). (C) OF caused a decrease in hepatic LCFA CoA levels, which were restored to SC levels by Scd1 ASO treatment (top left panel). Liver Scd1 deficiency in OF animals (black bars) resulted in a 3.5-fold increase in liver TG (top right panel) when compared with SCR ASO OF animals (gray bars). JNK phosphorylation and activity were markedly increased in OF Scd1-deficient animals as compared with OF control animals, while OF (gray bars) did not have any effect when compared with SC animals (white bars). Wet wt, wet weight. ${ }^{*} P<0.05$ versus SCR ASO OF.

compared with animals treated with scrambled ASO (SCR) (Figure 1B). The decrease in Scd1 activity was sufficient to alter the total pools of $\mathrm{C} 18$ acyl-CoAs, leading to a decrease in the C18 desaturation index, the ratio of C18:1 to C18:0 (oleyl-CoA/stearoyl-CoA) (Figure 1B). ASOs delivered i.p. were mainly taken up by the liver, but they also reached the peritoneal fat. Indeed, Scd1 mRNA levels were also decreased in epididymal fat pads. Food intake and body weight were not significantly different between the 2 treatment groups (Supplemental Table 1; supplemental material available online with this article; doi:10.1172/JCI26991DS1).

Effects of Scd1 deficiency on lipid metabolism. Consistent with observations in chronic models of SCD1 deficiency $(24,27,28)$, the levels of circulating triglycerides (TGs) were decreased by SCD1 ASO treatment (data not shown). However, the hepatic concentration of long-chain fatty acyl-CoAs (LCFA-CoAs) and of TGs was increased (Figure 1C). Consistent with these increases in tissue lipid levels, treatment with Scd1 ASO also activated the JNK pathway in the liver as manifested by increased JNK phosphorylation and activity (Figure 1C).
Since SCD1 deficiency has been previously shown to be associated with decreases in the hepatic expression of lipogenic genes, we next examined the hepatic expression of fatty acid synthase (FAS), acetyl-CoA carboxylase (ACC), and cholesterol hydroxylase $7 \alpha$ (CYP7A1) and the phosphorylation of AMP kinase (AMPK) and $\mathrm{ACC}$ at the completion of the insulin clamp procedure (Figure 2, $\mathrm{A}$ and $\mathrm{B}$ ) and under basal conditions (Figure 2, C and D). At the completion of the clamp studies, the short-term downregulation of Scd 1 expression had only marginal effects on these liver enzymes (Figure 2B). Under basal (overnight fast) conditions, treatment with Scd1 ASO moderately decreased the expression of FAS, ACC, and CYP7A1 (Figure 2D). These findings suggest that increased liver lipogenesis is not likely to account for the increase in liver TG and LCFA-CoA levels in rats treated with Scd1 ASO.

Scd1 is required for diet-induced insulin resistance in rats. In order to examine the impact of Scd1 deficiency on diet-induced insulin resistance, we next performed insulin clamp studies in OF rats (Figure 3A). Under conditions of physiological hyperinsulinemia, 
A

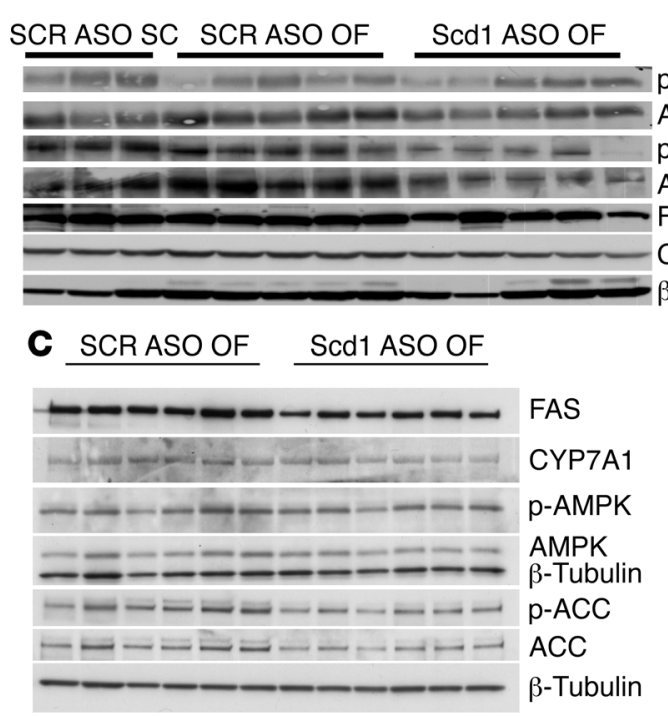

B

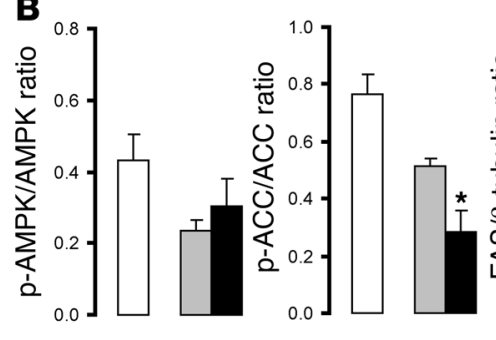

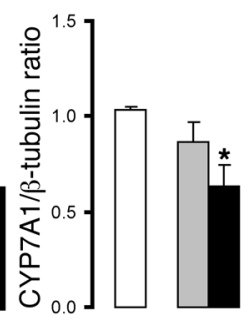

D
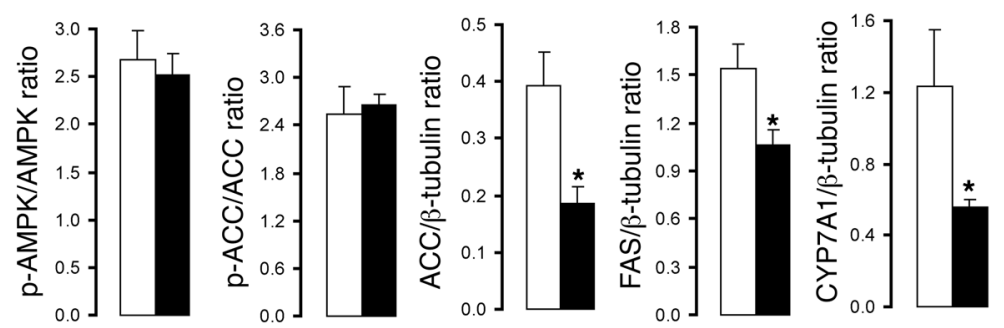

\section{Figure 2}

Scd1 deficiency moderately decreases the expression of lipogenic genes in the liver. (A and B) At the completion of insulin clamp studies, hepatic Scd1 deficiency in OF animals (black bars) did not significantly modify the levels of AMPK and FAS or the phosphorylation of AMPK and only modestly decreased ACC, p-ACC, and CYP7A1 in the liver when compared with SCR ASO OF (gray bars) and SC animals (white bars). (C and D) Under basal (overnight fast) conditions, hepatic Scd1 deficiency in OF animals (black bars) significantly decreased the expression of FAS, ACC, and CYP7A1 in the liver when compared with SCR ASO OF (gray bars) animals. ${ }^{*} P<0.05$ versus SCR ASO OF.

the rates of glucose infusion required to maintain euglycemia were markedly lower in OF rats compared with control rats fed a standard chow (SC) diet $(7.9 \pm 0.7$ versus $15.4 \pm 0.2 \mathrm{mg} / \mathrm{kg} / \mathrm{min} ; P<0.01)$ when SCR was administered (Figure 3B). On the other hand, when $\mathrm{Scd} 1 \mathrm{ASO}$ was given, the rate of glucose infusion required to maintain euglycemia in OF rats was markedly increased up to the levels observed in control SC rats $(15.2 \pm 0.4 \mathrm{mg} / \mathrm{kg} / \mathrm{min}$; Figure $3 \mathrm{~B})$. Scd1 ASO did not significantly alter the levels of glucoregulatory hormones (Supplemental Table 1) and the rate of glucose uptake (Figure 3B). Conversely, this intervention markedly suppressed liver glucose production (Figure 3, B and D), and this effect entirely accounted for its effect on glucose metabolism. Glucose production represents the net contribution of gluconeogenesis and glycogenolysis (Figure 3C). However, a portion of glucose entering the liver via phosphorylation of glucose is also a substrate for dephosphorylation via glucose-6-phosphatase (Glc-6-Pase, encoded in rodents by the G6pc gene), creating a futile (glucose) cycle (Figure 3C). To delineate the mechanisms by which Scd 1 inhibition modulates liver glucose homeostasis, we estimated the in vivo flux through Glc-6-Pase (Glc-6-Pase flux) and the relative contribution of glucose cycling, gluconeogenesis, and glycogenolysis to glucose output (Figure 3, D and E). Treatment with Scd1 ASO markedly and similarly decreased the rates of glucose cycling and Glc-6-Pase flux (Figure 3D) in parallel to its effects on glucose production (Figure 3D). The decrease in glucose production was accounted for by a marked inhibition of gluconeogenesis as well as glycogenolysis (Figure 3E and Supplemental Table 2).

Liver-selective Scd1 deficiency normalizes hepatic insulin action in $\mathrm{OF}$ rats. The i.p. delivery of Scd1 ASO led to a marked decrease in Scd1 expression not only in the liver but also in epididymal adipose tissue. In order to target Scd1 ASO to the liver, we delivered a 4-fold lower dose of ASO as a slow intraportal infusion rather than an i.p. bolus (Figure 4A). This protocol resulted in a significant decrease in liver expression of Scd1 in the absence of significant changes in Scd1 expression in epididymal fat (Figure 4B). Under conditions of physiological hyperinsulinemia, intraportal infusion of Scd1 ASO markedly improved insulin's ability to restrain glucose production (Figure 4B). To more directly examine whether the decreased expression of SCD1 in adipose tissue is required for the effects of SCD1 ASO treatment on liver glucose fluxes, we compared subgroups of OF rats displaying similar decreases in liver Scd1 expression induced by either i.p. or intraportal delivery of ASO (Figure 4, C-F). The i.p. administration of Scd 1 ASO dramatically suppressed Scd1 expression in epididymal fat pads while the intraportal infusion of the same ASO had no detectable impact on adipose tissue Scd1 expression (Figure 4, C and E). However, the 2 treatment regimens led to similar and dramatic improvements in hepatic insulin action (Figure 4, D and F). Thus, the approximately $70 \%$ decrease in liver Scd1 expression is sufficient to negate the onset of hepatic insulin resistance in this model.

Scd1 is required for diet-induced insulin resistance in mice. In order to examine whether Scd1 also plays a critical role in the development of diet-induced hepatic insulin resistance in HF-fed mice, we next performed insulin clamp studies in Scd1-deficient conscious mice (Figure 5, A-C). Treatment with Scd1 ASO markedly decreased hepatic Scd 1 mRNA and protein (Figure 5B). The enzymatic (desaturase) activity of Scd1 was markedly increased by HF feeding in mice and dramatically lowered by treatment with Scd1 ASO (Figure 5D). Under conditions of physiological hyperinsulinemia, the rates of glucose production were markedly increased in HF-fed mice compared with control mice fed an SC diet when the SCR was administered (Figure 5D). Importantly, in HF-fed mice treated with Scd1 ASO, the rate of glucose production was markedly suppressed to levels similar to those of control mice (Figure 5D). Of interest, 
A

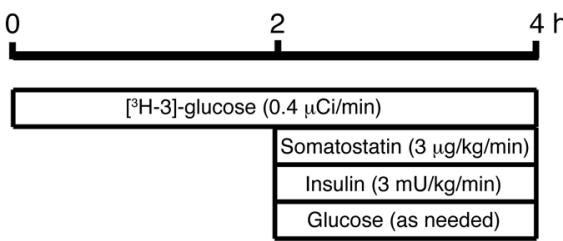

C

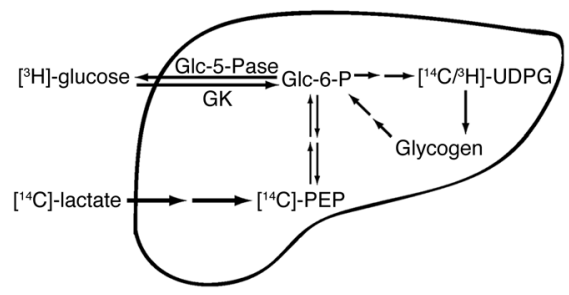

B Glucose

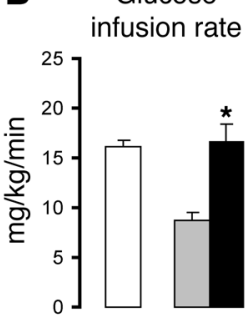

D

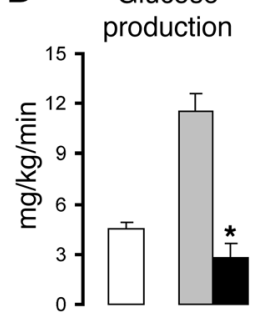

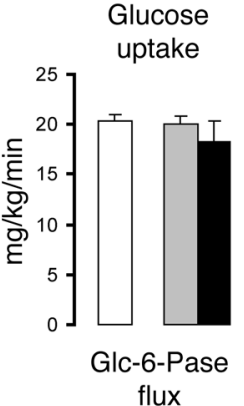

Glucose

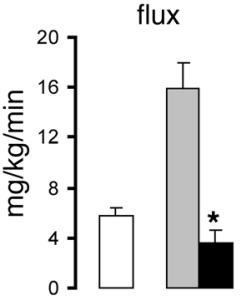

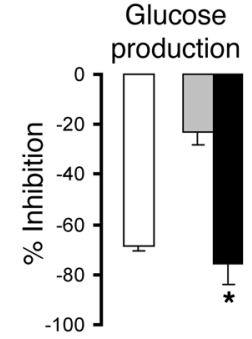

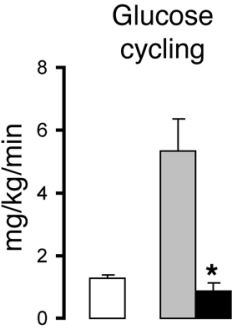

E Gluconeogenesis

Glycogenolysis
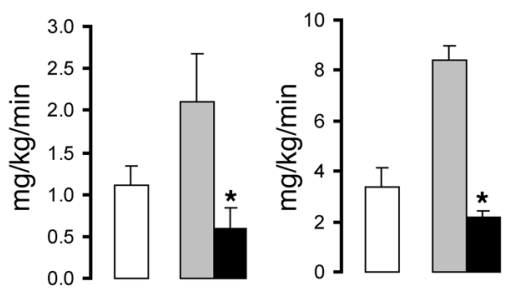

Figure 3

Scd1 deficiency negates the effects of OF on hepatic glucose fluxes. (A) Protocol for the hyperinsulinemic-pancreatic clamp procedure. (B) Glucose infusion rate, glucose uptake, and inhibition of glucose production (expressed as percentage inhibition from basal) during the clamp period in SC (white bars), control OF (gray bars), and Scd1-deficient OF animals (black bars). (C) Schematic of the major pathways and enzymatic reactions contributing to glucose production in the liver. $\left[{ }^{14} \mathrm{C}\right]-\mathrm{PEP},{ }^{14} \mathrm{C}$-phosphoenolpyruvate; ${ }^{3} \mathrm{H} /{ }^{14} \mathrm{C}$-UDPG, ${ }^{3 \mathrm{H}} /{ }^{14} \mathrm{C}$-UDP glucose; GK, glucokinase. (D) Glucose production, Glc-6-Pase flux, and glucose cycling during the clamp period in control SC (white bars), control OF (gray bars), and liver Scd1-deficient OF animals (black bars). (E) Rate of hepatic gluconeogenesis and glycogenolysis in control SC (white bars), control OF (gray bars), and liver Scd1-deficient OF animals (black bars). ${ }^{*} P<0.05$, versus SCR ASO OF; $n=5-6$ per condition.

Scd1 deficiency did not have an impact on the modest decrease in glucose uptake that was induced by HF feeding (Figure 5D). It should be noted that Scd1 ASO used in these experiments was directed against an entirely different region of Scd 1 mRNA. Overall, our findings indicate that $\mathrm{Scd} 1$ deficiency exerts a protective effect against the development of hepatic insulin resistance in HF-fed rats and mice.

Scd1 deficiency enhances Akt phosphorylation and suppresses phosphoenolpyruvate carboxykinase and Glc-6-Pase expression in OF rats. These metabolic data indicated that Scd1 ASO treatment enhanced insulin action to an extent that restored insulin sensitivity to normal levels in insulin-resistant animals. Enhanced insulin signaling could account for these effects. Thus, we next assessed the effect of shortterm Scd1 deficiency on Akt phosphorylation and on the expression of Glc-6-Pase, encoded by G6pc, and phosphoenolpyruvate carboxykinase (PEPCK), encoded by Pck1, in the liver. Decreased expression of Scd1 led to a marked increase in the hepatic phosphorylation of Akt. Western blot analysis (Figure 6A) showed increased phosphorylation of Akt at both Ser473 (4-fold; Figure 6B) and Thr308 (3-fold; Figure 6B) in animals treated with Scd1 ASO as compared with control (scrambled ASO [SCR]) ASO-treated animals. Hepatic Akt phosphorylation in these OF Scd1-deficient animals was even higher than in animals that are not insulin resistant on a regular chow diet. We next investigated whether these changes in Akt activation may have led to transcriptional modulation of the glucoregulatory enzymes PEPCK and Glc-6-Pase. Q-PCR experiments showed that the expression of PEPCK was suppressed by almost $90 \%$ in animals deficient in Scd1 as compared with control animals (Figure 6C). Similar measurements showed an even more dramatic effect on liver Glc-6-Pase, the expression of which was virtually abolished (Figure 6C) in Scd1 ASO-treated animals. Thus, it is likely that the decreases in in vivo Glc-6-Pase flux and gluconeogenesis are at least partly due to decreased hepatic expression of these 2 critical glucoregulatory enzymes.

Scd1 deficiency enhances hepatic insulin signaling in OF rats. In order to investigate the effect of Scd1 deficiency on early insulin signaling events, we next injected overnight-fasted rats with an i.p. bolus of insulin and sampled the livers 5 minutes later (Figure 7A). We assessed the effects of insulin on the phosphorylation of the insulin receptor (Figure 7, B and C) and insulin receptor substrate 1 (IRS1) (Figure 7, B and D) by immunoprecipitation followed by Western blots. We also examined the association of the $\mathrm{p} 85 \alpha$ subunit of PI3K with IRS1 (Figure 7, B and E). The acute administration of insulin failed to significantly stimulate the phosphorylation of IRS1 and its association with PI3K. However, treatment of OF rats with Scd1 ASO partly restored insulin responses. The pro- 

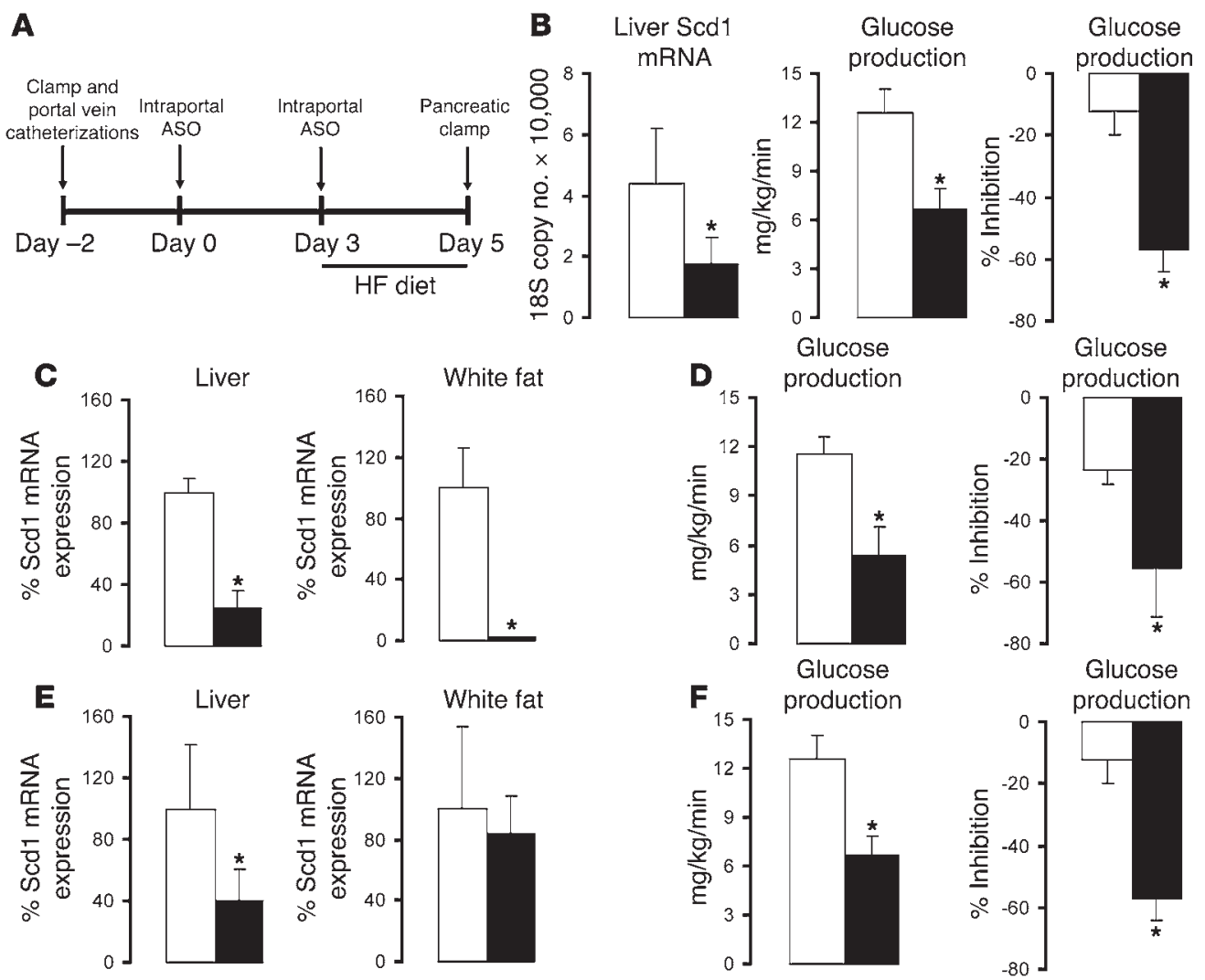

\section{Figure 4}

Selective attenuation of liver Scd1 expression normalizes hepatic insulin action in OF rats. (A) Protocol for the intraportal Scd1 ASO treatment (top panel). An indwelling catheter was placed into the portal vein, and 2 days later (day 0 ), the animals received a first intraportal injection of either SCR ASO or Scd1 ASO (25 mg/kg of body weight). On day 3, the rats were switched to a lard-enriched diet (HF) and received a second injection of ASOs. Lastly, on day 5, an insulin clamp procedure was performed (See Figure 2A). (B) Intraportal Scd1 ASO (black bar) decreased liver Scd1 mRNA when compared with SCR ASO (white bar; left panel). Selective attenuation of liver Scd1 (black bar) in OF rats improved hepatic insulin action when compared with SCR ASO (white bar) as shown by a significant decrease in glucose production (GP) (center panel) and a significant increase in insulin's ability to inhibit GP (right panel). (C-F) To examine whether the decreased expression of SCD1 in adipose tissue is required for the effects of Scd1 ASO on GP, we compared subgroups of OF rats displaying similar decreases in liver SCD1 induced by either i.p. (C and D) or intraportal (E and F) ASO. (C) i.p. Scd1 ASO markedly suppressed Scd1 expression in liver and white fat. (E) Intraportal Scd1 ASO markedly decreased Scd1 expression in the liver but not in adipose tissue. (D and F) The 2 treatment regimens led to similar improvements in hepatic insulin action. ${ }^{*} P<0.05$ versus SCR ASO OF; $n=5-6$.

tein expression of tyrosine phosphatase 1B (PTP1B) (Figure 7B) was only modestly decreased in response to Scd1 deficiency, and it may account in part for the increased phosphorylation of IRS1. These results suggest that the treatment of OF rats with Scd1 ASO resulted in a moderate stimulation of liver insulin signaling that could account in part for the increased phosphorylation of Akt.

\section{Discussion}

The signaling and metabolic effects of saturated and monounsaturated fatty acyl-CoAs are often distinct. For example, the inhibitory effect of LCFA-CoAs on ACC activity is greater for saturated than for monounsaturated fatty acyl-CoAs (29). The effects of SCD1derived monounsaturated fatty acyl-CoAs appear to be distinct from those of dietary fatty acids (30). Furthermore, there is evidence that increased availability of selective polyunsaturated LCFA-CoAs decreases the expression and activity of SCD1 in the liver (31).

SCD1 catalyzes the conversion of saturated to monounsaturated fatty acyl-CoAs. Here we asked whether SCD1 activity is required for the induction of hepatic insulin resistance by a diet enriched in saturated fatty acids. Although Scd1-deficient mice display improved glucose tolerance and insulin sensitivity $(24,27)$, these metabolic features could be secondary to the associated changes in energy balance, body composition, and lipid metabolism. Indeed, the chronic deficiency of SCD1 (either generalized or tissue-specific) per se leads to changes in body adiposity and energy expenditure $(24,27)$. These associated variables have a profound effect on insulin sensitivity. In the present study, we examined whether SCD1 expression is required for the development of diet-induced hepatic insulin resistance in the absence of the confounding effects of changes in body composition or energy expenditure $(24,27)$.

A reduction in SCD1 activity in liver was sufficient to restore normal insulin sensitivity in a model of diet-induced hepatic insulin resistance. Decreases in the rate of hepatic gluconeogenesis and glycogenolysis accounted for the lower glucose production in Scd1 ASO-treated rats. These decreases were accompanied by a dramatic reduction in the hepatic expression of the enzymes Glc-6-Pase and PEPCK. In addition, Scd1 deficiency markedly enhanced liver Akt phosphorylation at the completion of the insulin clamp studies. 
A
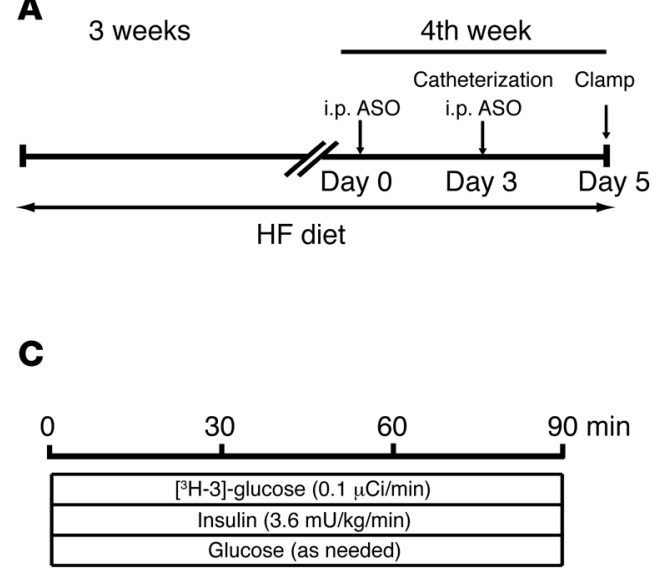
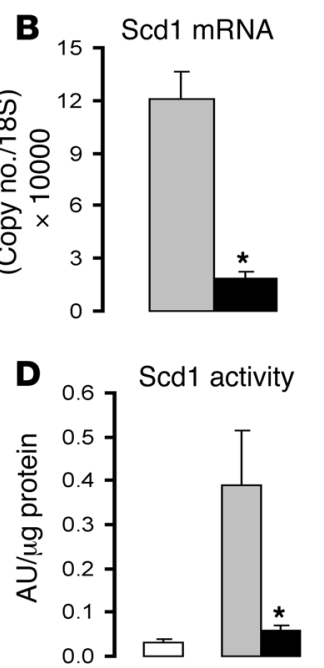
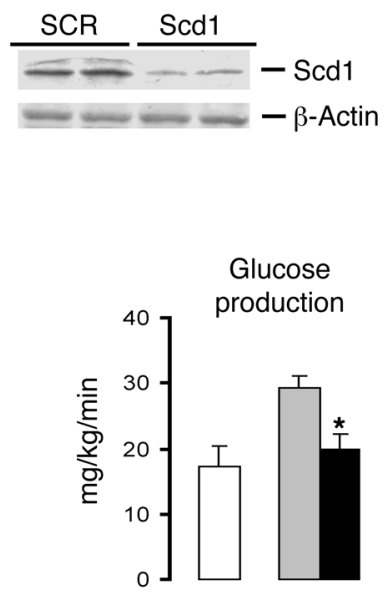

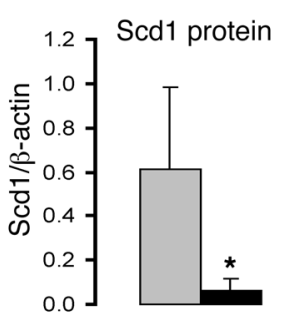

Glucose

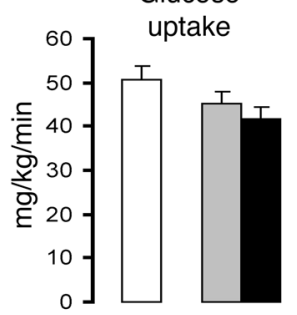

Figure 5

Scd1 deficiency normalizes hepatic insulin action in HF-fed mice. (A) Protocol for Scd1 ASO treatment in mice. Male C57BL/6J mice were allocated to a HF diet for 3 weeks. Five days before the completion of the protocol (day 0), the mice received an i.p. injection of either a SCR ASO or Scd1 ASO (100 mg/kg of body weight); on day 3, the animals received a second ASO injection, and vascular catheters were implanted; finally, on day 5, the mice were subjected to an insulin clamp procedure. (B) Scd1 ASO (black bars) markedly decreased the hepatic levels of Scd1 mRNA and protein compared with SCR ASO (grey bars) in HF diet-fed mice. (C) Protocol for the insulin clamp procedure in mice. (D) The enzymatic (desaturase) activity of Scd1 was markedly increased by HF feeding in mice (gray versus white bars) and dramatically lowered by treatment with Scd1 ASO (black bars). The rapid attenuation of liver Scd1 expression and activity prevented the development of hepatic insulin resistance induced by HF feeding in mice but did not modify insulin action on glucose uptake. ${ }^{*} P<0.05$ versus SCR ASO; $n=6-10$.

Consistent with observations in chronic models of SCD1 deficiency $(24,27,28)$, treatment with Scd1 ASO lowered the levels of circulating TGs. However, the hepatic concentration of LCFA-CoAs and of TGs was increased. This suggests that in the presence of short-term Scd1 deficiency, the rate of TG synthesis is not decreased at a time when TG secretion is already inhibited.

Marked stimulation of hepatic Akt activity can per se decrease blood glucose and insulin levels, induce fatty liver, and suppress the expression of Glc-6-Pase and PEPCK within just 4 days (32). Although these effects were elicited by adenovirus-mediated expression of a constitutively active Akt, leading to extreme activation of hepatic Akt, they are consistent with an important role of increased liver Akt activity in mediating the effects of SCD1 deficiency on hepatic glucose fluxes, on Glc-6-Pase and PEPCK expression, and on liver TGs (32). Of interest, a similar phenotype with decreased glucose production and increased liver TGs has also been reported following acute inhibition of Glc-6-Pase (33). Thus, the marked activation of Akt and the dramatic downregulation of Glc-6-Pase in the liver could account for the increase in liver TGs following short-term liver SCD1 deficiency. Regardless of the mechanism responsible for its accumulation, an increase in liver LCFA-CoAs and/or TGs is commonly associated with hepatic insulin resistance $(11,12,15)$ that is at least in part due to the activation of the JNK pathway $(17,34)$. Remarkably, treatment with SCD1 ASO markedly improved hepatic insulin signaling and action despite increased hepatic levels of TGs and LCFA-CoAs and activation of the JNK pathway. This dissociation between the effects of SCD1 deficiency on insulin signaling, glucose fluxes, and Glc-6-Pase/PEPCK gene transcription and its effects on body weight and liver lipids suggests a direct effect of SCD1 on hepatic insulin action. This finding appears to partly refute the causeeffect relationship between the phenotypic metabolic features of
SCD1 deficiency and the associated changes in lipid metabolism and body composition (24). There are previous examples in mice and rats in which an improvement in hepatic insulin action is associated with both decreased liver expression of Scd1 and increased hepatic levels of TGs $(32,33)$.

Our results support the notion that a decrease in Scd1 activity can per se improve insulin action and that this improvement can occur in the presence of increased hepatic stores of TGs. Thus, a primary decrease in liver Scd1 activity can prevent diet-induced hepatic insulin resistance in rats and mice. If the effects of SCD1 deficiency are confirmed in humans, the pharmacological inhibition of this enzyme should have independent and beneficial effects on both weight gain and insulin action. SCD1 deficiency may also account for the lack of hepatic insulin resistance in several genetic models in which a primary alteration in other steps in hepatic lipid metabolism also leads to a secondary decrease in SCD1 expression $(22,23,25)$.

\section{Methods}

Animals and experimental design: rat studies. Forty 10-week-old male SpragueDawley rats (Charles River Breeding Laboratories) were housed in individual cages and subjected to a standard light/dark cycle (lights on at 0600). One week before the pancreatic insulin clamp protocols (Figure 1B), catheters were placed in the right internal jugular veins and left carotid arteries as previously described $(35,36)$. Five and two days before the pancreatic clamp, rats received a single i.p. injection of either Scd1 or control (SCR) ASO (100 mg/kg of body weight). This dose of ASO was designed to achieve a robust downregulation of Scd1 expression in the liver with just 2 i.p. injections. Rats were fed an SC diet (Purina Mills Inc., Cat. No. 5001) until 3 days before the clamp; then the animals were switched to a highly palatable diet (Purina Mills Inc., Cat. No. 9389) generated by supplementation of the SC diet with $10 \%$ lard (Supplemental Table 3). An additional group 


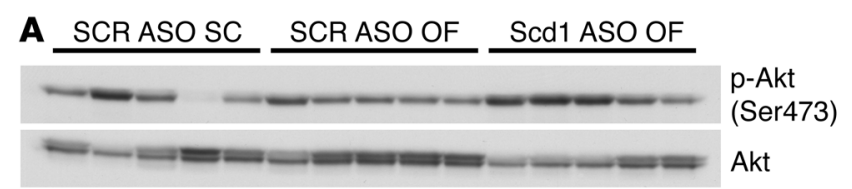

B
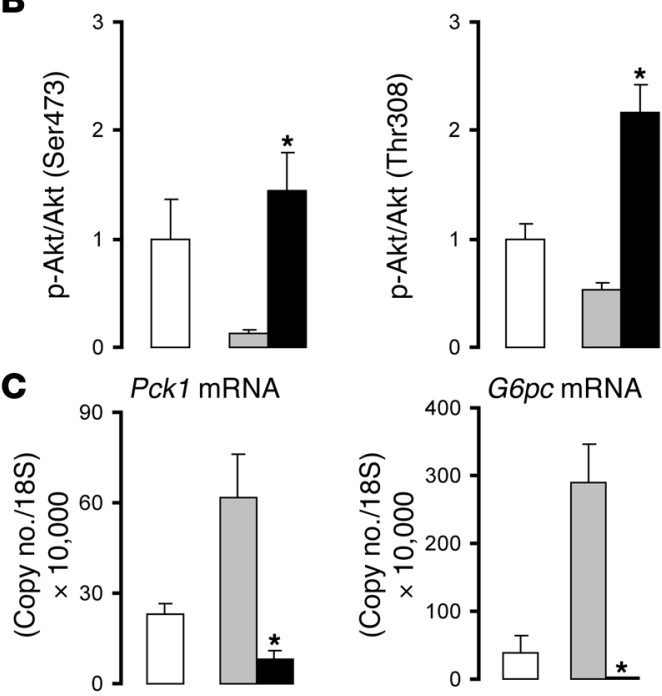

of animals fed on SC throughout the whole protocol of study received SCR ASO. To more selectively target the liver, we resorted to intraportal administration of ASOs (Figure 4A). In brief, another group of rats were subjected to the same protocol described above except that an additional intraportal catheter was placed 1 week before the insulin clamp protocol. ASOs were administered with the same schedule ( 2 injections) via the portal catheter at a dose of $25 \mathrm{mg} / \mathrm{kg}$ of body weight.

Animals and experimental design: mouse studies. Adult male C57BL/6J mice (27-32 g) were anesthetized with xylazine and ketamine and catheterized through the right internal jugular vein. The venous catheter was used for infusion, and blood samples were collected from the tail vein. Each animal was monitored for food intake and weight gain after surgery to ensure complete recovery. Mice were randomized in 3 groups. During the 3 weeks preceding the clamp study, 1 group received an SC diet (Purina Mills Inc.) from which $59 \%$ of calories were provided by carbohydrate, $28 \%$ by protein, and $12 \%$ by fat (Figure $5 \mathrm{~A}$ ). The remaining 2 groups received HF chow (Purina Mills Inc.) from which $45 \%$ of calories were provided by carbohydrate, $22 \%$ by protein, and $33 \%$ by fat). Thus, we studied 3 groups of mice: group 1 consisted of SC-fed mice treated with control ASO; group 2 consisted of HF-fed mice treated with control ASO; group 3 consisted of HF-fed mice treated with Scd1 ASO. All mice received 2 i.p. injections $(100 \mathrm{mg} / \mathrm{kg})$ of either control ASO (groups 1 and 2) or Scd1 ASO (group 3) during the week preceding the clamp study.

In vivo glucose kinetics and pancreatic insulin clamp procedure in rats. Pancreatic insulin clamp studies (Figure $3 \mathrm{~A}$ ) were performed as previously described to attain physiological hyperinsulinemia $(10,37)$. Rats were implanted with chronic catheters. At $t=0$, a primed, continuous infusion of HPLC-purified [ ${ }^{3} \mathrm{H}-3$ ]-glucose (PerkinElmer; $40 \mathrm{mCi}$ bolus, $0.4 \mathrm{mCi} / \mathrm{min}$ for duration of the study) was initiated and maintained throughout the 4 hours of the study. Samples for determination of ${ }^{3} \mathrm{H}$-glucose-specific activity were obtained at 10 -minute intervals throughout infusions. At $t=2$ hours, a pancreatic hyperinsulinemic-euglycemic clamp was initiated and maintained for the final 2 hours of the study. This procedure involved the infusion of somatostatin $(3 \mu \mathrm{g} / \mathrm{kg} / \mathrm{min})$ and insulin $(3 \mathrm{mU} / \mathrm{kg} / \mathrm{min})$

\section{Figure 6}

Scd1 deficiency enhances hepatic insulin action in OF rats. (A) Western blot analysis of Akt phosphorylation (Ser473) in the liver of SC control rats (SCR ASO SC) and in OF rats treated with either control (SCR ASO OF) or Scd1 ASO (Scd1 ASO OF). (B) OF (SCR ASO OF; gray bars) attenuated hepatic Akt phosphorylation at Ser473 and Thr308 as compared with SC diet (SCR ASO SC, white bars); Scd1 deficiency (Scd1 ASO OF, black bars) prevented the effect of OF and enhanced Akt phosphorylation 2- to 3-fold compared with animals on SC. (C) Liver expression of Pck1 and G6pc mRNA determined by Q-PCR. OF (gray bars) resulted in a marked increase in the hepatic expression of PEPCK and Glc-6-Pase when compared with an SC diet (white bars); ASO-mediated liver Scd1 deficiency (black bars) prevented the hepatic upregulation of these enzymes in response to OF and brought it down to levels below those of SC-fed animals. ${ }^{*} P<0.05$ versus SCR ASO OF.

and a variable infusion of $25 \%$ glucose periodically adjusted to clamp the plasma glucose concentration at approximately $8 \mathrm{mM}$. At the end of the in vivo studies, rats were anesthetized (pentobarbital, $55 \mathrm{mg} / \mathrm{kg}$ body weight, i.v.), and tissue samples were freeze clamped in situ with aluminum tongs precooled in liquid nitrogen. All tissue samples were stored at $-80^{\circ} \mathrm{C}$ for subsequent analysis.

In vivo glucose kinetics and pancreatic insulin clamp procedure in mice. Euglycemic clamps (Figure 5C) were performed in conscious, unrestrained, catheterized mice as previously described (38). Food was removed for 5 hours before beginning of in vivo studies. The infusion studies lasted a total of 90 minutes. A solution of glucose $(10 \% \mathrm{wt} / \mathrm{vol})$ was infused at a variable rate as required to maintain euglycemia $(8 \mathrm{mM})$. Mice received a constant infusion of HPLC-purified [ $\left.{ }^{3} \mathrm{H}-3\right]$-glucose $(0.1 \mu \mathrm{Ci} / \mathrm{min}$; PerkinElmer $)$ and insulin $(3.6 \mathrm{mU} / \mathrm{min} / \mathrm{kg}$ body weight). Thereafter, plasma samples were collected to determine glucose levels (at $t=10,20,30,40,50,60,70,80$, and 90 minutes) as well as $\left[{ }^{3} \mathrm{H}-3\right]$-glucose-specific activity (at $t=40,50,60,70,80$, and 90 minutes). Additionally, in 5 mice from each experimental group, 10 minutes before the end of the in vivo studies, $\left[\mathrm{U}-{ }^{14} \mathrm{C}\right]$-lactate $(5-\mu \mathrm{Ci}$ bolus, $0.4 \mu \mathrm{Ci} / \mathrm{min}$; PerkinElmer) was administered to determine the contribution of gluconeogenesis to the hepatic Glc-6-Pase pool. Steady state conditions for both plasma glucose concentration and specific activity were achieved by 40 minutes in these studies. At the end of the in vivo studies, mice were anesthetized (pentobarbital, $60 \mathrm{mg} / \mathrm{kg}$, i.v.), the abdomen was quickly opened, and adipose tissue and liver were freeze clamped in situ with aluminum tongs that were cooled in liquid nitrogen. The time between the injection of anesthesia and the freeze clamping of tissue samples was less than 60 seconds. Tissue samples were stored at $-80^{\circ} \mathrm{C}$ for further analysis.

Analytical procedures and calculations. Plasma glucose was measured by the glucose oxidase method (Glucose Analyzer II, Beckman Coulter). Plasma insulin and leptin, adiponectin, and glucagon levels were determined by RIA (Linco Research Inc.). Plasma nonesterified fatty acid concentrations were determined using an enzymatic method by an automated kit according to the manufacturer's specifications (Waco Pure Chemical Industries Ltd.). The radioactivity of $\left[{ }^{3} \mathrm{H}-3\right]$-glucose in plasma was measured from supernatants of $\mathrm{Ba}(\mathrm{OH})_{2}$ and from $\mathrm{ZnSO}_{4}$ precipitates (Somogyi procedure) after each was evaporated to dryness for the removal of tritiated water. Measurements of hepatic ${ }^{14} \mathrm{C}$-phosphoenolpyruvate and ${ }^{3} \mathrm{H} /{ }^{14} \mathrm{C}$-UDP glucose-specific activities were obtained from 2 sequential chromatographic separations, as previously described (39-42). Calculations were performed as described (43). In brief, the rates of ${ }^{4} \mathrm{C}$-phosphoenolpyruvate-gluconeogenesis, Glc-6-Pase flux, and glucose cycling (glucose cycling = Glc-6-Pase flux - glucose production) were calculated as previously described (43). Net glycogenolysis was calculated as the difference between glucose production and gluconeogenesis. 
A

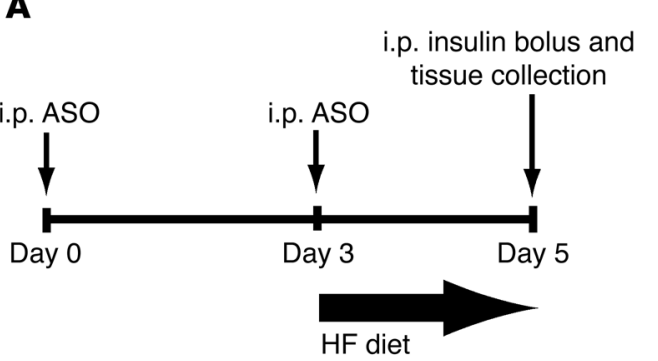

B

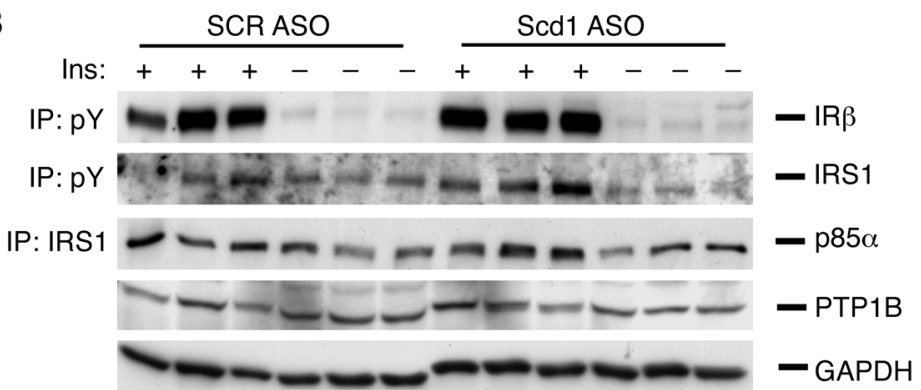

C
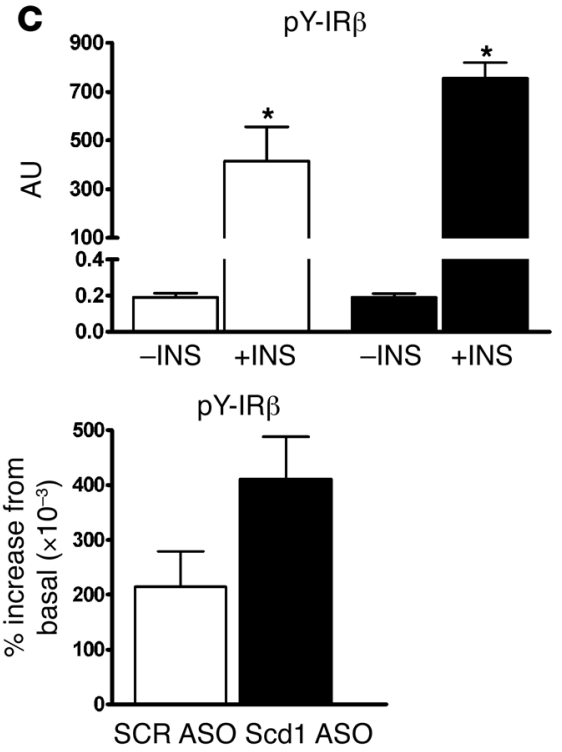

D
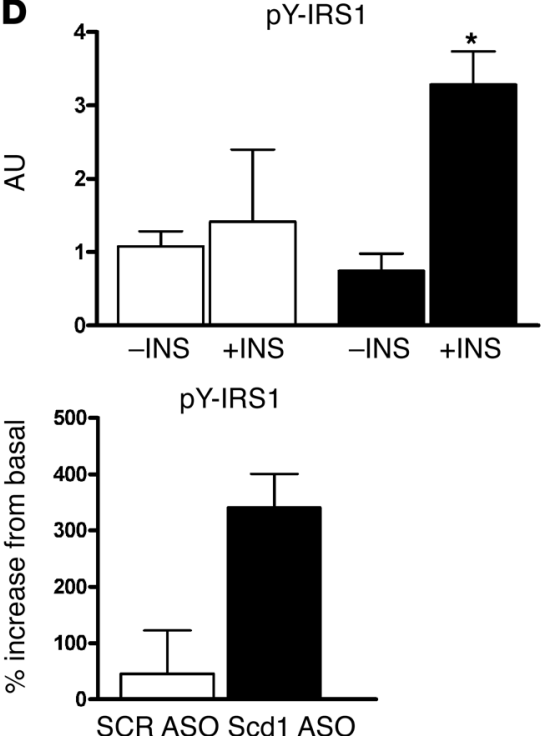

$\mathbf{E}$

E $\quad \mathrm{p} 85 \alpha$
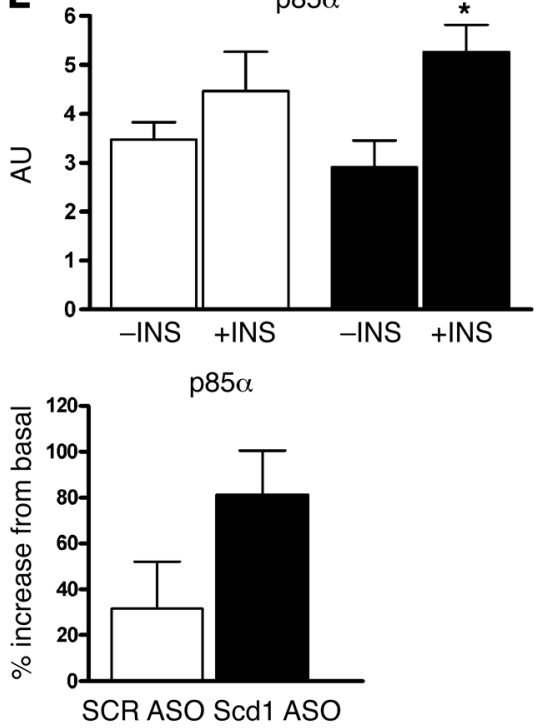

Figure 7

Scd1 deficiency enhances hepatic insulin signaling in OF rats. (A) To investigate the effect of Scd1 deficiency on early insulin signaling, we injected fasted rats with an i.p. bolus of insulin and sampled the livers 5 minutes later. The acute administration of insulin failed to stimulate the phosphorylation of Irs1 (B and D) and its association with PI3K (B and E). However, treatment of OF rats with Scd1 ASO partly restored insulin responses (B, D, and E). (B) Western blot analysis of immunoprecipitated tyrosine-phosphorylated IR $\beta$ and Irs1, Irs1-associated p85 $\alpha$ subunit of PI3K, and PTP1B in livers from control (SCR ASO) or Scd1 ASO-treated OF rats in the basal (-Ins) or insulin-stimulated state (+Ins). Densitometric analysis revealed a 35\% decrease in PTP1B protein in Scd1 ASO- compared with SCR ASO-treated livers (data not shown). (C) Densitometric analysis showed that insulin-stimulated tyrosine phosphorylation of IR $\beta$ was modestly increased by Scd1 deficiency. (D) Densitometric analysis showed that insulin-stimulated tyrosine phosphorylation of Irs1 was markedly enhanced by Scd1 deficiency. (E) The recruitment of $p 85 \alpha$ PI3K to Irs1 was also enhanced in livers from Scd1-deficient (black bars) when compared with SCR ASO (white bars) OF rats. In $\mathbf{C}$ and $\mathbf{D}$, left panels show basal and insulin-stimulated values while right panels show percentage increase from basal values. ${ }^{*} P<0.05$ versus SCR ASO OF; $n=3-6$ (average \pm SEM). pY, phosphotyrosine.

Scd1 activity. Liver Scd1 activity assays were performed essentially as described (26). At the end of incubation, long-chain acyl-CoAs were extracted and fractionated by reverse-phase HPLC as previously reported (44). Chromatographically resolved stearoyl-CoA (C18:0) and oleyl-CoA (C18:1) peaks were collected and counted in a $\beta$-scintillation counter. Protein was assayed by the bicinchoninic acid method using a commercially available kit (Pierce Biotechnology) according to the manufacturer's instructions.

Gene expression. Scd1, Glc-6-Pase (G6pc), and PEPCK (Pck1) mRNAs were measured by Q-PCR as described (37). In brief, total RNA was extracted from either liver or fat tissue using the TRIzol reagent (Invitrogen Corp.) according to the manufacturer's instructions. cDNA was then synthesized from $2 \mu \mathrm{g}$ of total RNA using the Superscript III First Strand kit (Invitrogen Corp.). Samples of RNA in which the reverse transcriptase was omitted (noRT controls) were also included. The cDNA and no-RT controls were diluted 1:20 with PCR grade water, and $2 \mu \mathrm{l}$ from each sample was used for PCR in a total volume of $20 \mu \mathrm{l}$. The FastStart DNA Master SYBR Green I kit (Roche
Applied Science) and a LightCycler 2 instrument (Roche Applied Science) were used in all experiments. The $18 \mathrm{~S}$ mRNA expression was routinely measured for normalization purposes. The primer sequences used were as follows: 5'-CTACAAGCCTGGCCTCCTGC-3' and 5'-GGACCCCAGGGAAACCAGGA-3' for Scd1; 5'-GCGACCGTCCCCTTTGCATCTGTC-3' and 5'-CCACCAAACACTCCCCCTCCTCC-3' for G6pc; 5'-GCCTCCTCAGCTGCATAATGGTCT-3' and 5'-GAATGCTTTCTCGAAGTCCTCTTCTG-3' for Pck1; and 5'-AGGGTTCGATTCCGGAGAGG-3' and 5'-CAACTTTAATATACGCTATTGG-3' for ribosomal 18S. The mRNA copy number was calculated from standard curves obtained using cloned target templates (plasmid DNA) of known copy number for each gene of interest.

LCFA-CoA. Liver LCFA-CoAs were extracted and measured as previously described (44).

TGs. Hepatic TGs were extracted by the Folch method (45). Liver and plasma TG were measured using a commercially available colorimetric assay kit (Sigma-Aldrich) according to the manufacturer's instructions. 
Western blot analysis. Liver tissue (50-100 mg) was homogenized in a detergent-based lysis buffer (M-PER; Pierce Biotechnology) supplemented with protease and phosphatase inhibitors. The extracts were incubated on ice for 20 minutes and then centrifuged at $15,000 \mathrm{~g}$ to remove tissue debris; the supernatants were saved and frozen at $-80^{\circ} \mathrm{C}$ until analysis. Then, $50 \mu \mathrm{g}$ of protein were heat denatured in SDS-PAGE sample buffer and resolved on denaturing (SDS) $10 \%$ polyacrylamide gels (SDS-PAGE). Fractionated proteins were electrophoretically transferred to nitrocellulose membranes by standard procedures (46). Membranes were immunoblotted with the appropriate primary antibodies; immune complexes were detected by ECL (Western Lightning, PerkinElmer) using horseradish peroxidase-conjugated secondary antibodies. Bands were imaged on $x$-ray film and digitized for quantification using ImageQuant software version 5.0 (GE Healthcare). $\beta$-tubulin, $\beta$-actin, or GAPDH was used to control for loading differences.

JNK activity assay. Liver JNK activity assay was performed using a kit from Cell Signaling Technology per the manufacturer's instructions. In brief, $200 \mu \mathrm{g}$ of liver protein were used to pull down JNK using immobilized glutathione-S-transferase-tagged c-Jun. After extensive washing, the immobilized affinity complexes were incubated 30 minutes at $37^{\circ} \mathrm{C}$ in the absence or presence of $200 \mu \mathrm{M}$ ATP. The reactions were stopped by the addition of Laemmli sample buffer and subsequent boiling. Phosphorylated c-Jun ( $\mathrm{p}-\mathrm{c}-\mathrm{Jun}$ ) was detected by Western blotting using $\mathrm{p}-\mathrm{c}$-Jun antibodies and ECL imaging.

Insulin signaling protocol. To examine early insulin signaling events in the liver, another group of OF rats were treated with either control (SCR) or Scd1 ASO as described in Animals and experimental design: rat studies. These animals were not subjected to pancreatic clamps but were instead challenged with an i.p. bolus of either insulin $(5 \mathrm{U} / \mathrm{kg})$ or saline after overnight fasting. Five minutes later, the animals were sacrificed and the liver was collected and processed immediately for analysis. About $300-500 \mathrm{mg}$ of liver was homogenized in 10 volumes of lysis buffer (50 mM HEPES, $\mathrm{pH}=7.7,100 \mathrm{mM}$ sodium pyrophosphate, $100 \mathrm{mM}$ sodium fluoride, $50 \mathrm{mM} \beta$-glycerophosphate, $10 \mathrm{mM}$ sodium orthovanadate, $10 \mathrm{mM}$ EDTA, 1\% NP40, $2 \mathrm{mM}$ PMSF, complete protease inhibitor cocktail; Roche Applied Science). The homogenates were incubated for 30 minutes on ice and then centrifuged at $3,800 \mathrm{~g}$ to remove tissue debris; the supernatants were used for the subsequent procedures. Protein concentration was measured by the bicinchoninic acid method described above, and protein concentration in all samples was adjusted to $20 \mathrm{mg} / \mathrm{ml}$. We incubated $16 \mathrm{mg}$ of total protein from each lysate with $10 \mu \mathrm{g}$ of either anti-IRS1 antibody (Upstate USA Inc.) or 4G10 phosphotyrosine antibody (Upstate USA Inc.) at $4^{\circ} \mathrm{C}$ for 2 hours. Next, $20 \mu \mathrm{l}$ of protein-A sepharose (for IRS1) or protein-G sepharose (for phosphotyrosine) beads (Amersham Biosciences) was added and incubated at $4^{\circ} \mathrm{C}$ for 1 hour. The beads were then washed 5 times with $1 \mathrm{ml}$ of lysis buffer and boiled with $60 \mu \mathrm{l}$ of Laemmli buffer. The immunoprecipitates were size fractionated by SDS-PAGE (10 $\mu \mathrm{l}$ of sample was applied in each lane), transferred to nitrocellulose as described above, and subjected to Western blot analysis.

Antibodies. Rabbit polyclonal or monoclonal antibodies to ACC, phospho-ACC (Ser79), Akt, p-Akt (Ser473 and Thr308), AMPK- $\alpha$, p-AMPK- $\alpha$ (Thr172), JNK, and p-SAPK/JNK (Thr183/Tyr185) were from Cell Signaling Technology; mouse monoclonal $\beta$-tubulin antibodies were obtained from Covance Research Products; FAS antibodies were from BD Biosciences - Pharmingen. The following were obtained from Santa Cruz Biotechnology Inc.: CYP7A1 rabbit polyclonal antibodies, insulin receptor $\beta$ subunit (IR $\beta$ ) antibodies, and mouse Scd1 goat polyclonal antibodies. IRS1 antibodies were from Upstate USA Inc., and p85 $\alpha$ subunit of PI3K antibodies were from Cell Signaling Technology. Rabbit polyclonal antibodies to PTP1B were obtained from Abcam, and GAPDH mouse monoclonal antibodies were from Research Diagnostics Inc.

Statistics. Statistical analysis was done using unpaired 2-tailed Student's $t$ test or analysis of variance. The study protocol was reviewed and approved by the Institutional Animal Care and Use Committee of the Albert Einstein College of Medicine.

\section{Acknowledgments}

We wish to thank Bing Liu, Clive Baveghems, Stanislaw Gaweda, and Yuhua Wang for expert technical assistance. H. Ono is the recipient of a fellowship from the Sankyo Foundation of Life Science. This work was supported by grants from the NIH (DK 48321, DK 45024, AG 21654, and DK 20541) to L. Rossetti.

Received for publication September 28, 2005, and accepted in revised form April 4, 2006.

Address correspondence to: Luciano Rossetti, Diabetes Research Center, Albert Einstein College of Medicine, 1300 Morris Park Avenue, New York, New York 10461, USA. Phone: (718) 430-4118; Fax: (718) 430-8557; E-mail: rossetti@aecom.yu.edu.
1. Ford, E.S., and Giles, W.H. 2003. A comparison of the prevalence of the metabolic syndrome using two proposed definitions. Diabetes Care. 26:575-581.

2. Kopelman, P.G., and Hitman, G.A. 1998. Diabetes. Exploding type II. Lancet. 352(Suppl. 4):SIV5.

3. Flegal, K.M., Carroll, M.D., Ogden, C.L., and Johnson, C.L. 2002. Prevalence and trends in obesity among US adults, 1999-2000. JAMA. 288:1723-1727.

4. Friedman, J.M. 2000. Obesity in the new millennium. Nature. 404:632-634

5. West, D.B., Boozer, C.N., Moody, D.L., and Atkinson, R.L. 1992. Dietary obesity in nine inbred mouse strains. Am. J. Physiol. 262:R1025-R1032.

6. West, D.B., Waguespack, J., and McCollister, S. 1995. Dietary obesity in the mouse: interaction of strain with diet composition. Am. J. Physiol. 268:R658-R665.

7. Schemmel, R., Mickelsen, O., and Gill, J.L. 1970. Dietary obesity in rats: body weight and body fat accretion in seven strains of rats. J. Nutr. 100:1041-1048.

8. Sclafani, A., and Springer, D. 1976. Dietary obesity in adult rats: similarities to hypothalamic and human obesity syndromes. Physiol. Behav. 17:461-471.

9. Kraegen, E.W., et al. 1991. Development of muscle insulin resistance after liver insulin resistance in high-fat-fed rats. Diabetes. 40:1397-1403.

10. Wang, J., et al. 2001. Overfeeding rapidly induces leptin and insulin resistance. Diabetes. 50:2786-2791.

11. Chen, M.T., Kaufman, L.N., Spennetta, T., and Shrago, E. 1992. Effects of high fat-feeding to rats on the interrelationship of body weight, plasma insulin, and fatty acyl-coenzyme A esters in liver and skeletal muscle. Metabolism. 41:564-569.

12. Griffin, M.E., et al. 1999. Free fatty acid-induced insulin resistance is associated with activation of protein kinase $\mathrm{C}$ theta and alterations in the insulin signaling cascade. Diabetes. 48:1270-1274.

13. Hegarty, B.D., Furler, S.M., Ye, J., Cooney, G.J., and Kraegen, E.W. 2003. The role of intramuscular lipid in insulin resistance. Acta Physiol. Scand. 178:373-383.

14. Jucker, B.M., Cline, G.W., Barucci, N., and Shulman, G.I. 1999. Differential effects of safflower oil versus fish oil feeding on insulin-stimulated glycogen synthesis, glycolysis, and pyruvate dehydrogenase flux in skeletal muscle: a $13 \mathrm{C}$ nuclear magnetic resonance study. Diabetes. 48:134-140.

15. Kraegen, E.W., Cooney, G.J., Ye, J.M., Thompson, A.L., and Furler, S.M. 2001. The role of lipids in the pathogenesis of muscle insulin resistance and beta cell failure in type II diabetes and obesity. Exp. Clin. Endocrinol. Diabetes. 109(Suppl. 2):S189-S201.
16. Petersen, K.F., Dufour, S., Befroy, D., Garcia, R., and Shulman, G.I. 2004. Impaired mitochondrial activity in the insulin-resistant offspring of patients with type 2 diabetes. N. Engl. J. Med. 350:664-671.

17. Samuel, V.T., et al. 2004. Mechanism of hepatic insulin resistance in non-alcoholic fatty liver disease. J. Biol. Chem. 279:32345-32353.

18. Shulman, G.I. 2000. Cellular mechanisms of insulin resistance. J. Clin. Invest. 106:171-176.

19. Yu, C., et al.2002. Mechanism by which fatty acids inhibit insulin activation of insulin receptor substrate-1 (IRS-1)-associated phosphatidylinositol 3-kinase activity in muscle. J. Biol. Chem. 277:50230-50236.

20. Chen, H.C., et al. 2002. Increased insulin and leptin sensitivity in mice lacking acyl CoA:diacylglycerol acyltransferase 1. J. Clin. Invest. 109:1049-1055. doi:10.1172/JCI200214672.

21. Kim, J.K., et al. 2004. Inactivation of fatty acid transport protein 1 prevents fat-induced insulin resistance in skeletal muscle. J. Clin. Invest. 113:756-763. doi:10.1172/JCI200418917.

22. Hammond, L.E., et al. 2005. Mitochondrial glycerol-3-phosphate acyltransferase- 1 is essential in liver for the metabolism of excess acyl-CoAs. J. Biol. Chem. 280:25629-25636. 
23. Neschen, S., et al. 2005. Prevention of hepatic steatosis and hepatic insulin resistance in mitochondrial acyl-CoA:glycerol-sn-3-phosphate acyltransferase 1 knockout mice. Cell Metab. 2:55-65.

24. Ntambi, J.M., et al. 2002. Loss of stearoyl-CoA desaturase- 1 function protects mice against adiposity. Proc. Natl. Acad. Sci. U. S. A. 99:11482-11486.

25. Maeda, K., et al. 2005. Adipocyte/macrophage fatty acid binding proteins control integrated metabolic responses in obesity and diabetes. Cell Metab. 1:107-119.

26. Cohen, P., et al. 2002. Role for stearoyl-CoA desaturase- 1 in leptin-mediated weight loss. Science. 297:240-243.

27. Jiang, G., et al. 2005. Prevention of obesity in mice by antisense oligonucleotide inhibitors of stearoylCoA desaturase-1. J. Clin. Invest. 115:1030-1038. doi:10.1172/JCI200523962.

28. Attie, A.D., et al. 2002. Relationship between stearoyl-CoA desaturase activity and plasma triglycerides in human and mouse hypertriglyceridemia. J. Lipid Res. 43:1899-1907.

29. Lunzer, M.A., Manning, J.A., and Ockner, R.K. 1977. Inhibition of rat liver acetyl coenzyme A carboxylase by long chain acyl coenzyme A and fatty acid. Modulation by fatty acid-binding protein. J. Biol. Chem. 252:5483-5487.

30. Miyazaki, M., Kim, H.J., Man, W.C., and Ntambi, J.M. 2001. Oleoyl-CoA is the major de novo product of stearoyl-CoA desaturase 1 gene isoform and substrate for the biosynthesis of the Harde- rian gland 1-alkyl-2,3-diacylglycerol. J. Biol. Chem. 276:39455-39461.

31. Ntambi, J.M. 1992. Dietary regulation of stearoylCoA desaturase 1 gene expression in mouse liver. J. Biol. Chem. 267:10925-10930.

32. Ono, H., et al.2003. Hepatic Akt activation induces marked hypoglycemia, hepatomegaly, and hypertriglyceridemia with sterol regulatory element binding protein involvement. Diabetes. 52:2905-2913.

33. Bandsma, R.H., et al. 2001. Acute inhibition of glucose-6-phosphate translocator activity leads to increased de novo lipogenesis and development of hepatic steatosis without affecting VLDL production in rats. Diabetes. 50:2591-2597.

34. Hirosumi, J., et al. 2002. A central role for JNK in obesity and insulin resistance. Nature. 420:333-336.

35. Liu, L., et al. 1998. Intracerebroventricular leptin regulates hepatic but not peripheral glucose fluxes. J. Biol. Chem. 273:31160-31167.

36. Obici, S., et al. 2001. Central melanocortin receptors regulate insulin action. J. Clin. Invest. 108:1079-1085. doi:10.1172/JCI200112954.

37. Gutierrez-Juarez, R., Obici, S., and Rossetti, L. 2004. Melanocortin-independent effects of leptin on hepatic glucose fluxes. J. Biol. Chem. 279:49704-49715.

38. Pocai, A., et al. 2005. Hypothalamic K(ATP) channels control hepatic glucose production. Nature. 434:1026-1031.

39. Giaccari, A., and Rossetti, L. 1992. Predominant role of gluconeogenesis in the hepatic glycogen repletion of diabetic rats. J. Clin. Invest. 89:36-45. 40. Giaccari, A., and Rossetti, L. 1989. Isocratic highperformance liquid chromatographic determination of the concentration and specific radioactivity of phosphoenolpyruvate and uridine diphosphate glucose in tissue extracts. J. Chromatogr. 497:69-78.

41. Rossetti, L., et al. 1993. Mechanism by which hyperglycemia inhibits hepatic glucose production in conscious rats. Implications for the pathophysiology of fasting hyperglycemia in diabetes. J. Clin. Invest. 92:1126-1134.

42. Rossetti, L., et al. 1996. Hepatic overexpression of insulin-like growth factor-II in adulthood increases basal and insulin-stimulated glucose disposal in conscious mice. J. Biol. Chem. 271:203-208.

43. Barzilai, N., et al. 1997. Leptin selectively decreases visceral adiposity and enhances insulin action. J. Clin. Invest. 100:3105-3110.

44. Woldegiorgis, G., Spennetta, T., Corkey, B.E., Williamson, J.R., and Shrago, E. 1985. Extraction of tissue long-chain acyl-CoA esters and measurement by reverse-phase high-performance liquid chromatography. Anal. Biochem. 150:8-12.

45. Folch, J., Lees, M., and Sloane Stanely, G.H. 1957. A simple method for the isolation and purification of total lipides from animal tissues. J. Biol. Chem. 226:497-509.

46. Towbin, H., Staehelin, T., and Gordon, J. 1979. Electrophoretic transfer of proteins from polyacrylamide gels to nitrocellulose sheets: procedure and some applications. Proc. Natl. Acad. Sci. U. S. A. 76:4350-4354. 